Methods: Retrospective longitudinal unicentric observational study. Clinical records of all patients diagnosed with SLE who had received treatment with belimumab were reviewed. Demographic characteristics, clinical manifestations and reason for belimumab indicaton were collected.

Results: The twelve patients included in the sample were women. Median age was 48.5 years $(31-70)$. The most frequent reason for indication of belimumab was uncontrolled arthritis. The average time of treatment with belimumab in the total sample was $27.5(+/-26.24)$ months, with a median of 12 months (4-78). Mean treatment time (cases in which belimumab was discontinued excluded) was $31.25(+/-26.98)$ months with a median of 25 (5-78). The average dose reduction of prednisone after initiation of treatment with belimumab (in patients in which it was considered effective) was $5 \mathrm{mg}$ per day (+/- 5). It should be noted that of the 14 cases, treatment was only discontinued in 4 patients, 2 of which were withdrawn due to ineffectiveness. There were 2 adverse events that required drug withdrawal: neutropenia and urothelial carcinoma. (Table). Conclusion: BLM is a well tolerated drug and effective in clinical practice. Adverse effects leading to drug withdrawal are infrequent.

Disclosure of Interests: Carolina Merino Argumánez: None declared, Olga Rusinovich: None declared, Consuelo Ramos Giráldez Speakers bureau: Sanofi, María Espinosa: None declared, Hilda Godoy: None declared, Carmen Barbadillo Mateos: None declared, Jose Campos Esteban: None declared, Mercedes Jiménez Palop: None declared, Jesus Sanz: None declared, Luis Fernando Villa Alcázar: None declared, Carlos Isasi Zaragoza : None declared, Monica Fernandez Castro: None declared, José Luis Andréu Sánchez: None declared

DOI: 10.1136/annrheumdis-2019-eular.7989

\section{AB0476 SPECIAL ASPECTS OF GLUCOCORTICOID THERAPY IN PATIENTSWHO TREATED WITH ANTI-B-CELL AND ANTI-BLYS THERAPY}

Anna Mesnyankina, Sergey Solovyev, Elena Aseeva, Evgeny Nasonov. Nasonova Research Institute of Rheumatology, Moscow, Russian Federation

Background: The basis of treatment of SLE are glucocorticoids (GCs), which since their introduction into clinical practice, have led to increased survival and reduced early mortality of SLE patients. However, the need to use high doses of GCs, as well as long-term use of medium doses to maintain disease remission, leads to the development of serious adverse reactions. This leads to an increase in the risk of irreversible organ damage. In this regard, it is important to search for ways to prevent the use of high doses of GCs, minimizing the dose of GCs.

Objectives: To assess special aspects and dynamics of oral glucocorticoid (GC) therapy in SLE patients treated with anti-B-cell and anti-BlyS therapy.

Methods: The study included 64 SLE pts (5M/58F), divided into 3 groups: Group I - 47 patients (SLEDAI2K 16[11;20] scores), receiving rituximab (RTX) i/v infusions by drop at 500 - $2000 \mathrm{mg}$ dose-range. Group II included 10 patients (SLEDAI2K 10[8;11] scores) treated with Belimumab (BLM) at $10 \mathrm{mg} / \mathrm{kg}$ once a month. The remaining 7 patients from Group III (SLEDAI2K 10[9;16] scores) were administered a combination of RTX and BLM. They started treatment with RTX 500 (2 patients) or $1000 \mathrm{mg}$ (5 patients) infusions, and 3 months later BLM at standard scheme of $10 \mathrm{mg} / \mathrm{kg}$ once a month was initiated for 8 months. SLICC damage index (DI) was documented at baseline - before initiation of RTX and BLM - in 26 out of 64 patients $(40 \%)$ with SLICC DI score > 1 (1 - 5 scores); 20 patients out of them were administered RTX.

Results: 47 patients on RTX therapy received different oral GCs doses: high GCs doses (Me 40[30;50]mg/day) were documented in 11 (24\%) patients, moderate doses (Me 13[10;20]mg/day) - in 29 (61\%) patients, and low doses (Me 5[5;7,5]mg/day) - in 7 (15\%) patients. Patients on BLM and combination therapy were administered GCs at doses $\leq 20 \mathrm{mg}$ (Me $15[5 ; 20] \mathrm{mg} /$ day and Me $8,75[5 ; 15] \mathrm{mg} /$ day, respectively)

During first 3 month of treatment GCs doses in all 3 Groups remained unmodified. By Mo $625 \%$ reduction in oral GCs doses was documented in: patients on RTX - 20[15;20]mg/day, 10[8,75;10]mg/day, 5[5;5] mg/day (respectively, in the groups with initially high, moderate and low GCs doses); BLM 10[7,5;10] mg/day, combination therapy $8,75[5 ; 15] \mathrm{mg} /$ day. By Mo $12 \mathrm{Me}$ GCs dose in all 3 Groups did not exceed $10 \mathrm{mg} / \mathrm{day}$. In view of SLE exacerbations in patients from Group I (RTX) additional RTX infusions at Mo 6, 9 and 12 were administered in $8(20 \%)$ patients. Figure 1 presents the dynamics of oral GCs doses in patients from Group I (RTX), who were divided in 3 subgroups based on baseline (high, moderate or low) GCs dose, and also dynamics of GCs dose in patients from Groups II (BLM) and III (RTX+BLM).

Increase in SLICC score by Mo 12 of follow up was documented in patients on RTX therapy with baseline pre-existing organ damage (5 patients). There was no increase in SLICC scores in BLM and RTX +BLM treatment groups.

Conclusion: Combination therapy results in achieving rapid control of SLE activity thanks to RTX effects, and the combination with BLM allows significantly prolongs this result, minimizing the risk of exacerbation. And a very specific gain from combination RTX+BLM therapy is a chance to manage patients on moderate-to-low oral GCs doses, therefore, reducing the risk of irreversible organ damage. Increasing organ damage score in RTX Group is most likely associated with intake of higher GCs doses.

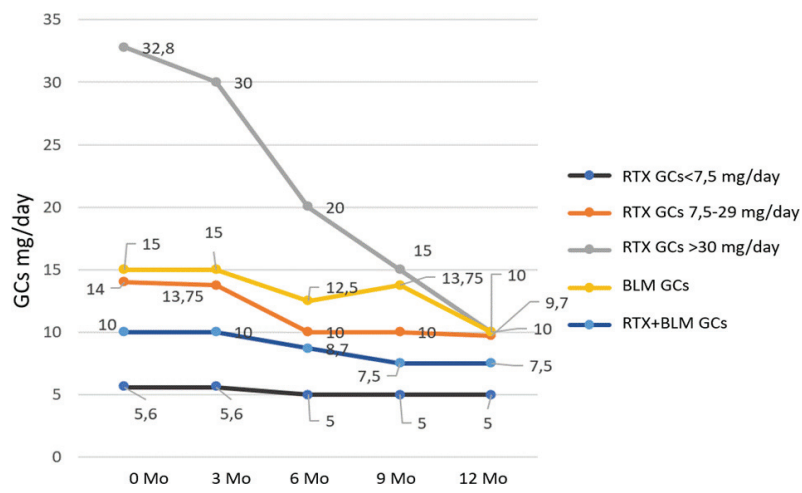

Figure 1. The dose of oral GCs in patients treated with RTX, BLM and combined treatment, $\mathrm{Me}$

Disclosure of Interests: Anna Mesnyankina: None declared, Sergey Solovyev: None declared, Elena Aseeva: None declared, Evgeny Nasonov Speakers bureau: Pfizer, Inc., MSD, Novartis, AbbVie Inc., Celgen Corporation, Biocad, Janssen, UCB, Inc.

DOI: 10.1136/annrheumdis-2019-eular.4294

\section{AB0477 THE EFFECT OF CLOSTRIDIUM BUTYRICUM ON INTESTINAL FLORA OF PATIENTS WITH SYSTEMIC LUPUS ERYTHEMATOSUS ZHANG MINGXING**1 ${ }^{1}$ XU- FANG YIN ${ }^{1}$, XIAO-FENG LI ${ }^{2}$}

zhang mingxing ${ }^{1}$, Xu-Fang Yin ${ }^{1}$, LI Xiao-Feng ${ }^{2} .{ }^{1} 2$ The Second Hospital of Shanx Medical University, Tai Yuan, China; ${ }^{2}$ The Second Hospital of Shanxi Medical University, Taiyuan, China Objectives: To investigate the effect of Clostridium butyricum on intestinal flora in patients with systemic lupus erythematosus.

Methods: Forty-four patients with systemic lupus erythematosus who were randomly selected from our hospital were given the oral administration of Clostridium butyricum live capsule $840 \mathrm{mg}$ twice a day. The concentration of methane and hydrogen at each time point before and after treatment for 44 patients was detected and compared by hydrogen and methane in lactulose breath test (LBT).

Results: After 28 days of treatment with Clostridium butyricum capsules, there was no significantly statistical difference in exhaled hydrogen concentration or methane concentration between $0 \mathrm{~min}, 30 \mathrm{~min}, 60 \mathrm{~min}$ and $90 \mathrm{~min}$ before and after treatment. See Table 1.

Conclusion: Numerous studies have shown that taking probiotics can promote the growth of normal flora and reduce the reproduction of other abnormal flora, which has a certain impact on the structure and quantity of There was no significantly statistical difference in exhaled hydrogen concentration or methane concentration at various points in time between the 44 patients before and after treatment, suggesting that there was no significant change in the structure and quantity of intestinal flora.

intestinal flora, which may be resulted from our low dose Clostridium butyricum live capsules or short treatment time, or need to be combined with other probiotics. These hypothesis above need us to further explore, in general, using microbes as a therapeutic target spot to regulate the illness of SLE by interfering with intestinal flora through diet, probiotics or fecal transplantation provides a promising prospect for clinical treatment of SLE.

Table 1. Methane and hydrogen concentrations before and after intervention by the experimenter[PPM,Md(P25,P75)]

\begin{tabular}{lccc}
\hline & $\begin{array}{c}\text { before } \\
\text { intervention }\end{array}$ & $\begin{array}{c}\text { after } \\
\text { intervention }\end{array}$ & $p$ \\
\hline H2(0min) & $16(8.5,30)$ & $21(5.5,35)$ & 0.697 \\
H2(30min) & $27(13,45)$ & $27(6,61)$ & 0.872 \\
H2(60min) & $27(11,49.5)$ & $21(5.5,58.5)$ & 0.977
\end{tabular}




$\begin{array}{lccc}\mathrm{H} 2(90 \mathrm{~min}) & 27(14,59) & 27(11.5,55) & 0.600 \\ \mathrm{CH} 4(0 \mathrm{~min}) & 4(3,5) & 4(3,5.5) & 0.699 \\ \mathrm{CH} 4(30 \mathrm{~min}) & 5(4,6) & 5(3.5,7) & 0.776 \\ \mathrm{CH} 4(60 \mathrm{~min}) & 5(4,5) & 5(3.3,6) & 0.057 \\ \mathrm{CH} 4(90 \mathrm{~min}) & 5(4,5) & 5(4,6.5) & 0.142 \\ \mathrm{H} 2+\mathrm{CH} 4(0 \mathrm{~min}) & 20(12.5,35.5) & 26(9,39) & 0.747 \\ \mathrm{H} 2+\mathrm{CH} 4 & 32(19,50) & 32(12,67) & 0.872 \\ (30 \mathrm{~min}) & & & \\ \mathrm{H} 2+\mathrm{CH} 4 & 31(17,54) & 30(13.5,64.5) & 0.909 \\ (60 \mathrm{~min}) & & & \\ \mathrm{H} 2+\mathrm{CH} 4 & 31(20,63.5) & 31(19,60) & 0.000\end{array}$

(90min)

Using Wilcoxon test, $\mathrm{P}<0.05$

Disclosure of Interests: None declared

DOI: 10.1136/annrheumdis-2019-eular.6647

\section{AB0478 PREGNANCY OUTCOME IN WOMEN WITH SYSTEMIC LUPUS ERYTHEMATOSUS TREATED WITH HYDROXYCHLOROQUINE: 10 YEARS EXPERIENCE}

Kyaw Min Tun, Rahana Rahman, Ixora Kamisan @ Atan, Ani Amelia Zainuddin, Ruslinda Mustafar, Mohd Shahrir Mohamed Said. Universiti Kebangsaan Malaysia Kuala Lumpur Campus, Kuala Lumpur, Malaysia

Background: Pregnancy in SLE patients is closely associated with the usage of hydroxychloroquine [1]

Objectives: To determine pregnancy outcomes in women with Systemic Lupus Erythematosus who were treated with hydroxychloroquine in Universiti Kebangsaan Malaysia Medical Centre.

Methods: Pregnant women with Systemic Lupus Erythematosus who had antenatal follow up and delivery in Universiti Kebangsaan Malaysia Medical Centre between $1^{\text {st }}$ January 2007 and $1^{\text {st }}$ January 2017 were retrospectively analyzed. Data collection was done via medical case notes and laboratory investigations. Study population was categorised into two groups based on hydroxychloroquine treatment during pregnancy. Incomplete pregnancy records were excluded.

Results: There were 82 completed pregnancies included with 47 (57.3\%) in $\mathrm{HCQ}$ group and $35(42.7 \%)$ in non $\mathrm{HCQ}$ group. Amongst HCQ users, there were significantly more pregnancies with musculoskeletal involvement $(p=0.03)$, heavier mean neonatal birth weight $(p=0.02)$ and prolonged duration of pregnancy $(p=0.001)$. In non-HCQ users, the rate of recurrent miscarriages $(p=0.003)$, incidence of hypertension $(p=0.01)$ and gestational diabetes mellitus $(p=0.01)$ and concurrent medical illness $(p=0.005)$ were significantly more. Hydroxychloroquine use during pregnancy was protective against hypertension $(p=0.001)$ and the gestational age at delivery had significant effect on the neonatal birth weight $(p=0.001)$. However, duration of the disease had significant negative effect on the neonatal birth weight $(p=0.016)$.

Conclusion: $\mathrm{HCQ}$ enhance better neonatal outcomes and reduce adverse pregnancy outcome and antenatal complications such as hypertension and diabetes.

\section{REFERENCES}

[1] M Leroux, C Desveaux, M Parcevaux. Impact of hydroxychloroquine on preterm delivery and intrauterine growth restriction in pregnant women with systemic lupus erythematosus: a descriptive cohort study. Lupus 2015. https://doi.org/10.1177/0961203315591027

Disclosure of Interests: Kyaw Min Tun: None declared, Rahana Rahman: None declared, Ixora Kamisan @ Atan: None declared, Ani Amelia Zainuddin: None declared, Ruslinda Mustafar: None declared, Mohd Shahrir Mohamed Said Paid instructor for: Eli Lilly, Speakers bureau: Eli Lilly DOI: 10.1136/annrheumdis-2019-eular.578

\section{AB0479 RITUXIMAB MAY BE BENEFICIAL IN THE TREATMENT OF SEVERE FORMS OF SYSTEMIC AUTOIMMUNE DISEASES REFRACTORY TO STANDARD IMMUNOSUPPRESSIVE THERAPY. NINE-YEARS EXPERIENCE FROM SINGLE CLINICAL CENTRE}

Marta Olejárova ${ }^{1,2}$, Veronika Balajková ${ }^{1,2}{ }^{1} 1$ st Medical Faculty, Charles University, Clinic of Rheumatology, Prague, Czech Republic; ${ }^{2}$ Institute of Rheumatology, Prague, Czech Republic

Background: Rituximab (RTX) is anti-CD20 monoclonal antibody, registered for the treatment of rheumatoid arthritis (RA). It has been used in clinical practice "of label" in various other conditions including systemic autoimmune diseases - systemic lupus erythematosus (SLE), systemic scleroderma (SScl), Sjögren's syndrome (SjS), polymyositis (PM) and dermatomyositis (DM) and ANCA associated vasculitides, but the results of clinical studies are inconherent. Nevertheless, RTX is frequently used in patients with severe forms of systemic autoimmune diseases with organ involvement after failure of conventional immunosupressive treatment. In Czech Republic, RTX may be used "of label" upon a special request in these special cases.

Objectives: To evaluate indications, clinical characteristics, general efficacy, safety and the impact of RTX therapy in patients with systemic autoimmune diseases treated in the Institute of Rheumatology, Praque since 2009 (a retrospective study).

Methods: The medical reports of all patients treated with RTX for other diagnosis than RA were analyzed and the clinical data (diagnosis, previous treatment with glucocorticosteroids and immunosuppressive drugs, number of RTX infusion, side effects and global evaluation of the efficacy by physician) were collected and analyzed.

Results: In the years 2009 - 2018, 50 patients with various systemic autoimmune diseases were treated with RTX in our clinic (SLE: $n=8$, SjS: $n=2$, SScl: $n=16, P M: n=6, D M: n=3$, MCTD: $=1$, ANCA associated vasculitides: $n=11$, overlap syndromes: $n=3$ ). Mean age of the group was $48,12 \pm 12,9$ years at the first RTX treatment and all of the patients experienced previous failure of treatment with 1-6 immunosuppressants The total number of RTX infusions was 208 with mean 5,32 applications per patient. We have followed the patients for 37,1 months (mean), rang ing from 2-114 months. During the follow-up two patients died due to complications of the disease, two patients were lost from the follow-up. The treatment was effective in majority of patients $(n=27)$ and global efficacy was evaluated as "excellent" in 2, "very good" in 10 and "good" in 15 patients. In seven patients the treatment had no effect and in 4 patients the effect could not be evaluated due to short time follow-up. The treatment was well tolerated by patients and we have experienced 39 moderate or severe infections (requiring antibiotics or hospitalization) mostly in patients with ANCA associated vasculitides. In several patients, mild decrease of $\mathrm{IgG}$ levels following the RTX treatment was observed but only in one patient required a subsequent substitution with immunoglobulines. We have not observed any malignancy in the group. The detailed characteristics and results of the patients by diagnosis are provided.

Conclusion: Rixuximab may be beneficial in some patients with severe forms of systemic autoimmune diseases refractory to the standard immunosuppressive therapy. The treatment is generally well tolerated and the infections are the most common side effect.

Acknowledgement: This study was supported by the Research Project No. 0002372801 of the Czech Ministry of Health.

Disclosure of Interests: None declared

DOI: 10.1136/annrheumdis-2019-eular.8276

\section{AB0480 PROLONGED CLINICAL REMISSION AND LOW DISEASE ACTIVITY STATUSES ARE ASSOCIATED WITH BETTER QUALITY OF LIFE IN SYSTEMIC LUPUS ERYTHEMATOSUS}

Nareerat Poomsalood, Sumapa Chaiamnuay, Pongthorn Narongroeknawin, Paijit Asavatanabodee, Rattapol Pakchotanon. Phramongkutklao Hospital and College of Medicine, Bangkok, Thailand

\section{Background:}

Treat to target (T2T) strategies in systemic lupus erythematosus (SLE) have been developed in order to control disease activity, improve healthrelated quality of life (HRQoL), reduce organ damage and decrease mortality. Previous studies revealed that both remission and low disease activity (LDA) had comparable damage accrual as well as mortality. However, studies of HRQoL in these two targets are few and there has been no comparative study between them:

Objectives: To determine the association between disease activity status (DAS) and HRQoL in SLE patients.

Methods: SLE patients in out-patient clinic during the previous 12 months were included in the study. Systemic Lupus Erythematosus-specific Quality-of-Life questionnaire (SLEQoL) was measured at the last visit. DAS was determined retrospectively during the previous year. Three categories of DAS were defined: clinical remission (CR): clinical quiescent disease according to Systemic Lupus Erythematosus Disease Activity Index 2000 (SLEDAI-2K), prednisolone < $5 \mathrm{mg} /$ day; low disease activity (LDA): SLE DAI-2K (without serological domain) $\leq 2$, prednisolone $<7.5 \mathrm{mg} /$ day; and non-optimally controlled status: those who were not in CR/LDA. Immuno suppressive drugs (maintenance dose) and antimalarials were allowed. Both CR and LDA have been maintained for at least 1 year. The 